\title{
The Little Bang: Searching for quark-gluon matter in relativistic heavy-ion collisions
}

\author{
Ulrich Heinz*
}

Theoretical Physics Division, CERN, CH-1211 Geneva 23, Switzerland

I review the status of the search for quark-gluon plasma in relativistic heavy-ion collisions. The available data provide strong evidence for the "three pillars of the Little Bang model": strong radial expansion of the collision fireball with Hubble-like scaling, thermal hadron emission and primordial hadrosynthesis. It is argued that the initial state of the reaction zone exhibits features which cannot be understood with conventional hadronic dynamics, but are consistent with the formation of deconfined quark-gluon matter.

\section{PROLOGUE}

On Feb. 10, 2000, CERN announced officially [1] that (I paraphrase) "compelling evidence now exists for the formation of a new state of matter at energy densities about 20 times larger than that in the center of atomic nuclei and temperatures about 100000 times higher than in the center of the sun. This state exhibits characteristic properties which cannot be understood with conventional hadronic dynamics but which are qualitatively consistent with expectations from the formation of a state of matter in which quarks and gluons no longer feel the constraints of color confinement." I will here explain the scientific arguments [2] which led to this conclusion. The announcement was not triggered by a single experimental discovery which had just happened, but emerged from a painstaking analysis of many different sets of data collected during 15 years of heavy-ion collision experiments at the CERN SPS, especially of results obtained during the last five years with the $158 \mathrm{AGeV} / \mathrm{c}^{207} \mathrm{~Pb}$ beam and reported over the last 24 months [3]. It happened at a time when the Relativistic Heavy Ion Collider RHIC at BNL was about to turn on and CERN was taking stock of what had been achieved so far with the up to then highest energy heavy-ion beams available. The announcement gave credit to the achievements of an international community of almost 500 enthusiastic physicists from all over the world who had initiated and driven to success the heavy-ion program at CERN, participating in seven large and several smaller experiments with complementary goals in order to search for the creation of the theoretically predicted "quark-gluon plasma" (QGP) in heavy-ion collisions.

The reaction of the international press to this announcement was very strong but the reports were not always accurate. Several stated that CERN had claimed the "discovery of quark-gluon plasma". This was not true: The CERN press release [1] and the scientific

\footnotetext{
*On leave from Institut für Theoretische Physik, Universität Regensburg, D-93040 Regensburg, Germany. Work supported in part by DFG, GSI, and BMBF. Email address: ulrich.heinz@cern.ch
} 
document on which it was based [2] had been formulated very carefully and made a clear and conscious distinction between "evidence for a new state of matter" (which we claimed) and "discovery of QGP" (which we didn't). I will also report on the remaining open questions, trying to point out (as I did in my talk on Feb. 10 [4]) what is needed to turn the present evidence into incontrovertible proof. Several crucial issues can only be resolved by heavy-ion experiments at the higher energies provided by RHIC and LHC.

\section{THE QUARK-HADRON TRANSITION}

The QCD phase diagram [5] features a transition from a gas of hadronic resonances (HG) at low energy densities to a quark-gluon plasma (QGP) at high energy densities. The critical energy density $\epsilon_{\mathrm{c}}$ is of the order of $1 \mathrm{GeV} / \mathrm{fm}^{3}$. It can be reached by either heating matter at zero net baryon density to a temperature of about $T_{\mathrm{c}} \approx 170 \mathrm{MeV}$, or by compressing cold nuclear matter to baryon densities of about $\rho_{\mathrm{c}} \sim 3-10 \rho_{0}$ (where $\rho_{0}=0.15 \mathrm{fm}^{-3}$ is the equilibrium density), or by combinations thereof. A simple version of the phase diagram is shown in Figure 1.

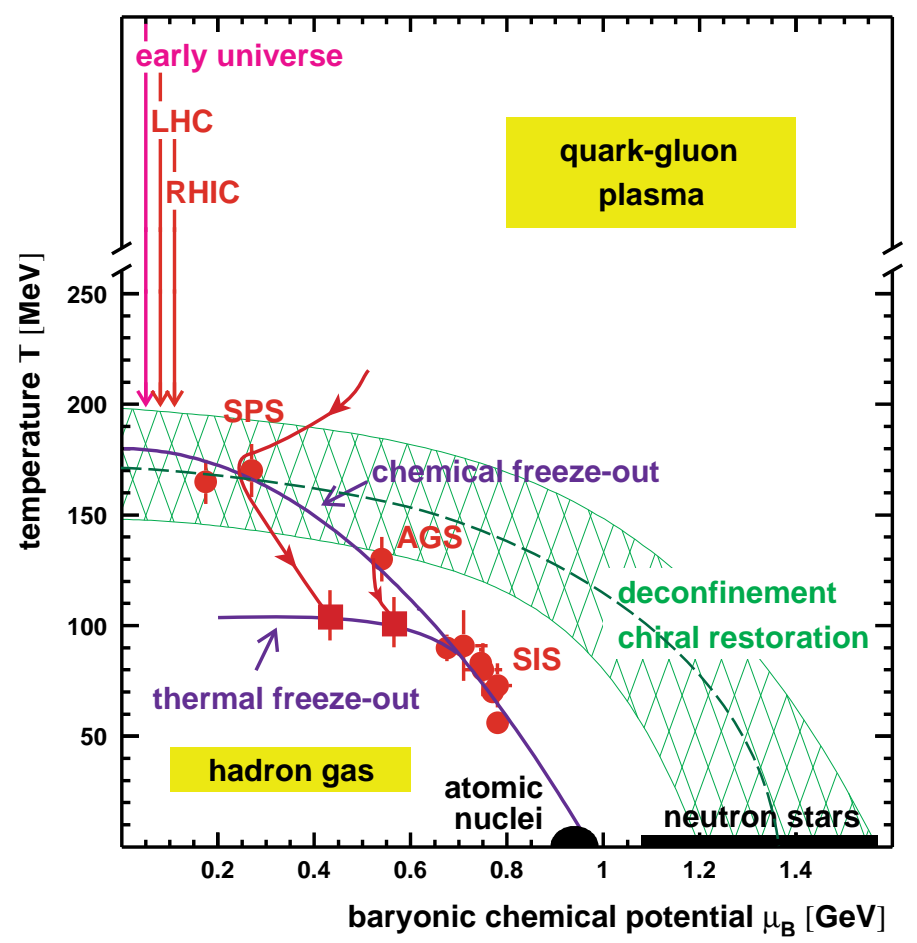

Figure 1. Sketch of the QCD phase diagram, temperature $T$ vs. the baryon chemical potential $\mu_{\mathrm{B}}$ associated with net baryon density $\rho_{\mathrm{B}}$. The cross-hatched region indicates the expected phase transition and its present theoretical uncertainty, with the dashed line representing its most likely location. Lines with arrows indicate expansion trajectories of thermalized matter created in different environments. (The lines labelled "RHIC", "LHC" and "early universe" should be much closer to the temperature axis but would then have been difficult to draw.) For a discussion of the chemical and thermal freeze-out lines and the location of the data points see text.

By colliding heavy ions at high energies, one hopes to heat and compress hadronic matter to energy densities above $\epsilon_{\mathrm{c}}$. At lower beam energies (SIS @ $1 \mathrm{AGeV/c}$ ), the nuclei are stopped and the nuclear matter is compressed and moderately heated. At higher beam energies one reaches higher temperatures, but since the colliding nuclei are no longer completely stopped, the baryon chemical potential of the matter created at rest in the center-of-momentum system decreases (AGS @ $10 A \mathrm{GeV} / c$ and SPS @ $160 A \mathrm{GeV} / c$ ). At the heavy ion colliders RHIC $(\sqrt{s}=200 \mathrm{AGeV})$ and LHC $(\sqrt{s}=5500 \mathrm{AGeV})$ the baryon chemical potential of the reaction fireball is so small that one essentially simulates baryon-free hadronic matter, very much like the expanding early universe. If the matter 
thermalizes quickly at energy densities above $\epsilon_{\mathrm{c}}$, it will pass through the quark-hadron phase transition as the collision fireball expands and cools.

Along the temperature axis at $\mu_{\mathrm{B}}=0$ our knowledge of the QCD phase diagram is based on hard theory (lattice QCD), but for nonzero baryon density we must rely on models interpolating between low-density hadronic matter, described by low-energy effective theories, and high-density quark-gluon plasma, described by perturbative QCD. The theoretical uncertainties at high-baryon densities are thus difficult to quantify and relatively large (typically $\mathcal{O}(30-50 \%)$ ). At zero baryon density, the situation is much cleaner: numerical simulations of QCD with 3 dynamical light quark flavors on the lattice are now available, and the systematic errors due to lattice discretization and continuum extrapolation are controllable and beginning to get small [6]. The critical temperature $T_{\mathrm{c}}$ for real-life QCD is predicted as $T_{\mathrm{c}} \approx 170 \mathrm{MeV} \pm 15 \%$ [6].7]. Near $T_{\mathrm{c}}$ the energy density in units of $T^{4}$ changes dramatically by more than a factor of 10 within a very narrow temperature interval (see Figure 2). Above $T \simeq 1.2 T_{\mathrm{c}}, \epsilon / T^{4}$ appears to settle at about $80 \%$ of the Stefan-Boltzmann value for an ideal gas of non-interacting quarks and gluons.

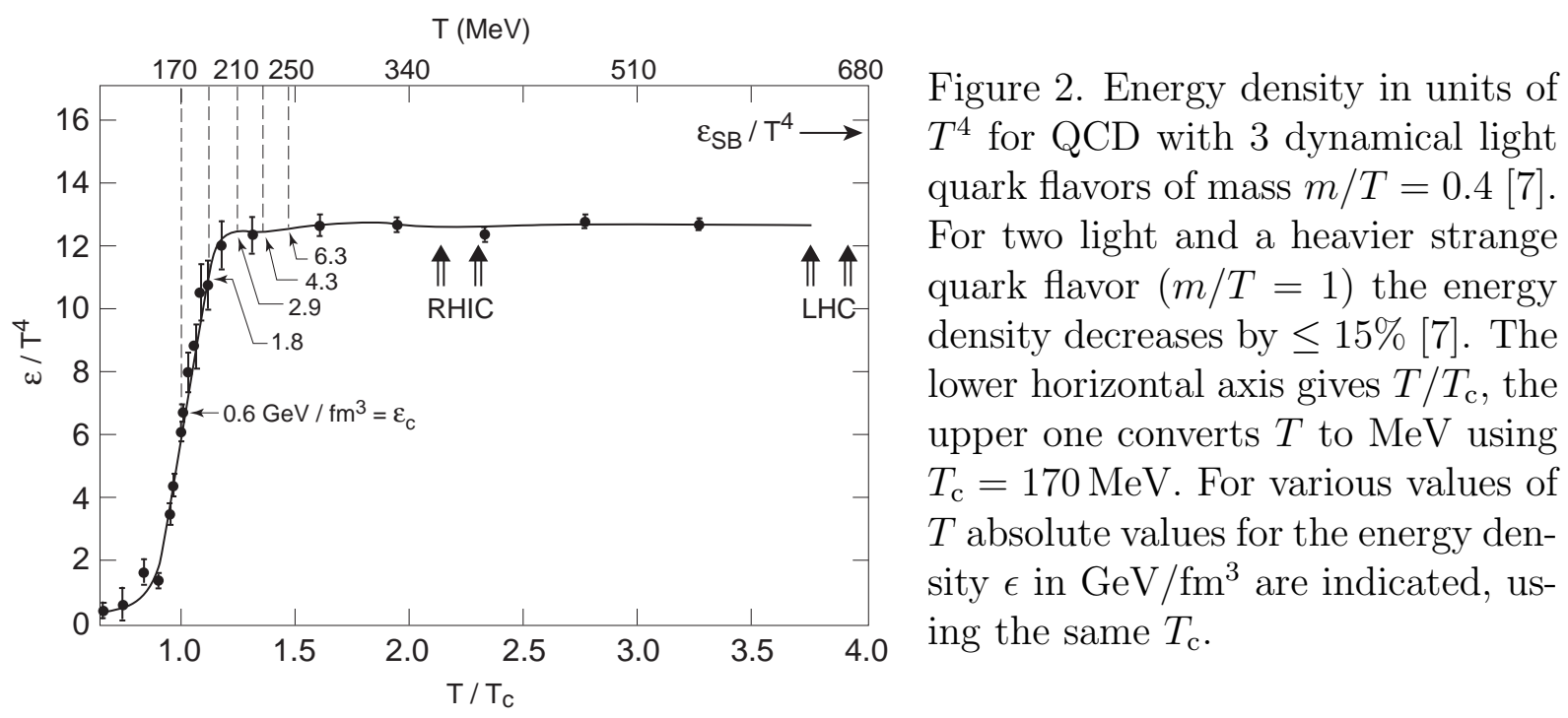

According to Figure 2 only about $600 \mathrm{MeV} / \mathrm{fm}^{3}$ of energy density are needed to make the transition to deconfined quark-gluon matter. It is, however, very expensive to reach temperatures well above $T_{\mathrm{c}}$ : an initial temperature of $220 \mathrm{MeV}$, about $30 \%$ above $T_{\mathrm{c}}$, already requires an initial energy density $\epsilon \simeq 3.5 \mathrm{GeV} / \mathrm{fm}^{3}$, about 6 times the critical value. We will see that this severely limits the reach of the CERN SPS into the new QGP phase: only the region at and slightly above $T_{\mathrm{c}}$ can be probed. We are "living at the edge" (W.Zajc)! To really penetrate deep into the new phase requires the much higher energy densities which become accessible with the heavy-ion colliders RHIC and LHC.

At $T_{\mathrm{c}}$ two phenomena happen simultaneously [8]: color confinement is broken, i.e. colored degrees of freedom can propagate over distances much larger than the size of a hadron, and the approximate chiral symmetry of QCD, which is spontaneously broken at low temperatures and densities, gets restored. Both effects are important since they significantly accelerate particle production: the liberation of gluons in large densities opens up new gluonic production channels, and the threshold for quark-antiquark pair 
production is lowered since the quarks shed a large fraction of their constituent mass which is dynamically generated by their interaction with the quark condensate characterizing the spontaneously broken chiral symmetry at low densities.

Much less important for the dynamics of heavy-ion collisions is the actual order of the phase transition: depending on the value of the strange quark mass, existing lattice calculations allow for anything between a smooth crossover, similar to the one seen in Figure 2, and a weakly first order transition with a latent heat which is small on the vertical scale of Figure 2. The resulting differences in the dynamical evolution of the expanding fireball are almost certainly unmeasurable, except if the phase diagram features a critical point and the experiments pass close to it [5].

\section{RECONSTRUCTING THE LITTLE BANG}

As the two nuclei hit each other with full speed, a superposition of nucleon-nucleon (NN) collisions occurs. What is different compared to individual NN collisions is that (i) each nucleon may scatter several times, and (ii) the liberated partons from different NN collisions rescatter with each other even before hadronization, as do the secondary hadrons produced in different NN collision. Both features change the particle production per participating nucleon. But only the rescattering processes can lead to a state of local thermal equilibrium, by redistributing the energy lost by the beams into the statistically most probable configuration. These rescatterings result in thermodynamic pressure acting against the outside vacuum which causes the reaction zone to expand collectively. The expansion cools and dilutes the fireball below the critical energy density of the quark-hadron transition, at which point hadrons are formed from the quarks and gluons (hadronization). Further interactions between these hadrons cease once their average distance exceeds the range of the strong interactions: the hadrons "freeze out".

The strong interactions among the partons and hadrons before freeze-out wipe out most information about their original production processes. The extraction of information about the interesting hot and dense early collision stage thus requires to exploit features which are either established early and not changed by the rescattering and collective expansion or can be reliably back-extrapolated. Correspondingly one classifies the observables into two classes, early and late signatures. (A comprehensive review of QGP signatures with a complete list of references can be found in [9].)

The conceptually cleanest early signatures are the directly produced real and virtual photons (i.e. not those resulting from hadron decays after freeze-out) since photons show no strong interaction and directly escape from the fireball (virtual photons materialize as $e^{+} e^{-}$or $\mu^{+} \mu^{-}$pairs). They are emitted throughout the expansion, but their production is expected to be strongly weighted towards the hot and dense initial stages. Unfortunately, direct photons are rare, and the experimental background from hadronic decay photons after freeze-out is enormous.

Another early signature are hadrons made of charmed quarks. At SPS energies, $c \bar{c}$ pairs can only be created in the primary NN collisions, because the secondary scatterings are not energetic enough to overcome the $c \bar{c}$ threshold. The only thing that can happen to them is a redistribution in phase-space, changing the relative amounts of mesons with hidden ( $c \bar{c}$ charmonium) and open charm $(\bar{c} q$ and $\bar{q} c)$. It was shown that such a redistribution is 
much easier in the color-deconfined QGP phase than by reinteraction of charmed particles with other hadrons; in this way charm-redistribution becomes also an early signature. But also charm production is a very rare process at SPS energies and below.

Hadrons made of up, down and strange quarks, which can be relatively easily produced and destroyed in all stages of the fireball expansion (I'll slightly qualify this statement further below with regard to strange hadrons), are late signatures. They provide only indirect information about the early collision stages. But they are very abundant, forming more than $99.99 \%$ of all emitted particles, and can thus be measured very accurately. We'll thus use these late signals to reconstruct the Little Bang and then check the consistency of the resulting picture with the less detailed direct information from the early signals.

I would like to note that this procedure is quite analogous to the reconstruction of the cosmological Big Bang from observations. The three observational pillars of the Big Bang theory are the Hubble expansion (which goes on until today), the cosmic microwave background of thermal photons (which decoupled when our universe was about 300,000 years old), and the measured abundances of light atomic nuclei (which reflect the primordial nucleosynthesis until about 3 minutes after the Big Bang). Almost everything before that time is based on a theoretical back-extrapolation using Einstein's equations and the Standard Model of particle physics and not on direct astrophysical observations. The only exception is the observed baryon number asymmetry which was presumably established very early but which is still not entirely understood.

\section{INITIAL CONDITIONS}

Before starting to analyze the final freeze-out stage of the collision using the late signatures, it is useful to get an idea about the global conditions of the fireball formed shortly after impact. Following Bjorken [10] it is possible to estimate the initially produced energy density by measuring the total transverse energy $E_{T}$ (excluding the fraction due to motion along the beam direction) and putting it into an estimated initial volume of the reaction zone. Assuming boost-invariant longitudinal expansion (which is expected to hold at high energies [10 and for which evidence exists in the midrapidity region even at SPS energies [11]) we can identify the space-time rapidity $\eta=0.5 \ln [(t+z) /(t-z)]$ with the momentum-space rapidity $y=0.5 \ln \left[\left(E+p_{z}\right) /\left(E-p_{z}\right)\right]$ and write [10]

$\epsilon_{\mathrm{Bj}}\left(\tau_{0}\right)=\frac{1}{\pi R_{\mathrm{rms}}^{2}} \frac{1}{2 \tau_{0}} \frac{d E_{T}}{d y}$.

Inserting $\pi R_{\mathrm{rms}}^{2}=63 \mathrm{fm}^{2}$ for the overlap area of two $\mathrm{Pb}$ nuclei colliding at zero impact parameter, choosing $\tau_{0}=1 \mathrm{fm} / c$ to evaluate the length $2 \tau d y=2 \tau d \eta$ of a slice of a cylinder of width $d \eta$ at midrapidity $y=\eta=0$, and using $d E_{T} / d y(y=0) \approx 400 \mathrm{GeV}$ for central $\mathrm{Pb}+\mathrm{Pb}$ collisions [12] ("very" central collisions even give up to $10 \%$ more) one obtains

$\epsilon_{\mathrm{Bj}}^{\mathrm{Pb}+\mathrm{Pb}}(1 \mathrm{fm} / c)=3.2 \pm 0.3 \mathrm{GeV} / \mathrm{fm}^{3}$.

As QGP searchers we thus play in the right ball-park; if the matter were already thermalized after $1 \mathrm{fm} / c$ (which at this point we don't know yet), Figure 2 tells us that the initial temperature would have been $T_{0} \simeq 210-220 \mathrm{MeV}$. Similar initial energy densities are obtained from a detailed phase-space analysis of the hadronic freeze-out state after 
back-extrapolation to the time before the onset of transverse expansion [13]. Perhaps more importantly, initial energy densities between 2 and $10 \mathrm{GeV} / \mathrm{fm}^{3}$ during the first 1-2 $\mathrm{fm} / c$ are obtained [14] even in approaches which avoid quarks and gluons as relevant degrees of freedom, like the URQMD code which uses hadronic strings and resonances to describe particle production and rescattering and is tuned to NN data. Indeed, for $\mathrm{Pb}+\mathrm{Pb}$ collisions at the SPS, URQMD predicts that the prehadronic component (strings) dominates over the produced hadrons for nearly $8 \mathrm{fm} / c$ [14]! This further strengthens our expectation to see new physics at the SPS.

\section{THERMAL FREEZE-OUT: AN EXPLODING THERMAL FIREBALL}

The measured hadron spectra contain two pieces of information: (i) Their normalization, i.e. the yields and abundance ratios, provides the chemical composition of the fireball at the "chemical freeze-out" point (i.e. when the hadron abundances freeze out); this yields information in particular about the degree of chemical equilibration, to be discussed in Section 7. (ii) The hadronic momentum spectra provide information about thermalization of the momentum distributions and collective flow. The latter is caused by thermodynamic pressure (resulting from intense rescattering among the consituents) and thus reflects, in a time-integrated way, the equation of state of the fireball matter. The expansion rate at "thermal freeze-out" (i.e. along the last-scattering hypersurface which marks the decoupling of the momenta) provides the Little Bang analogue of the Hubble constant for the Big Bang, while the corresponding freeze-out temperature parallels the temperature of the cosmic microwave background at the point of photon decoupling.

As in cosmology, where the photon temperature is affected by the Hubble expansion and red-shifted from originally $3000 \mathrm{~K}$ to $2.7 \mathrm{~K}$ today, the hadronic momentum spectra are affected by the collective expansion of the collision fireball. Only in this case the expansion occurs in three dimensions, and the fireball is observed from outside, resulting in a blue-shift of the apparent temperature of the spectra. Since the longitudinal expansion is ambiguous in that it is difficult to assess which fraction of the finally observed longitudinal flow is generated by hydrodynamic pressure and how much is a result of incomplete stopping of the two nuclei, we concentrate on transverse flow, reflected in the transverse mass $\left(m_{\perp}=\sqrt{m^{2}+p_{\perp}^{2}}\right)$ spectra. This type of flow is only created after impact.

Roughly, if rescattering among the fireball constituents results in thermalization and collective flow, the shapes of all hadronic $m_{\perp}$-spectra can be characterized by just two numbers: the temperature $T_{\mathrm{f}}$ and the mean transverse flow velocity $\left\langle v_{\perp}\right\rangle$ at freeze-out. More exactly, this is only true if all hadrons decouple simultaneously (i.e. their rescattering cross sections are similar), and the form in which the spectra are characterized by $\left\langle v_{\perp}\right\rangle$ may depend on the flow velocity and density profiles. For this presentation I will neglect the latter subtlety; the former can be checked experimentally.

In the relativistic region, i.e. for $m_{\perp}>2 m_{0}$, the rest masses can be neglected, and the effect of flow on the spectra is given by the simple blue-shift formula 15

$T_{\text {slope }}=T_{\mathrm{f}} \sqrt{\frac{1+\left\langle v_{\perp}\right\rangle}{1-\left\langle v_{\perp}\right\rangle}}$.

At large $m_{\perp}$ all hadron spectra should have the same inverse slope $T_{\text {slope }}$ (this tests ther- 
malization) but measuring it does not allow to separate thermal from collective motion.

In the nonrelativistic region $m_{\perp}<2 m_{0}$, on the other, flow does couple to the rest mass: for a linear transverse flow velocity profile and a Gaussian transverse density profile one finds exactly [16,17]

$T_{\text {slope }}=T_{\mathrm{f}}+\frac{1}{2} m_{0}\left\langle v_{\perp}\right\rangle^{2}$.

Such an approximately linear rest mass dependence is indeed observed. Figure 3 shows clearly that the spectra contain a collective flow component; inverse slopes of $300 \mathrm{MeV}$ or more as seen e.g. for the protons can obviously not be interpreted as hadronic temperatures (see Figures 1 and 2). There is some scatter between the data from different experiments, partly due to different kinematic regions for fitting the slope. The pion spectra are particularly troublesome due to the strong deformation at low $m_{\perp}$ from resonance decay contributions and Coulomb effects. Strictly speaking, the pions should not be included in this plot since they are never non-relativistic in the accessible region.

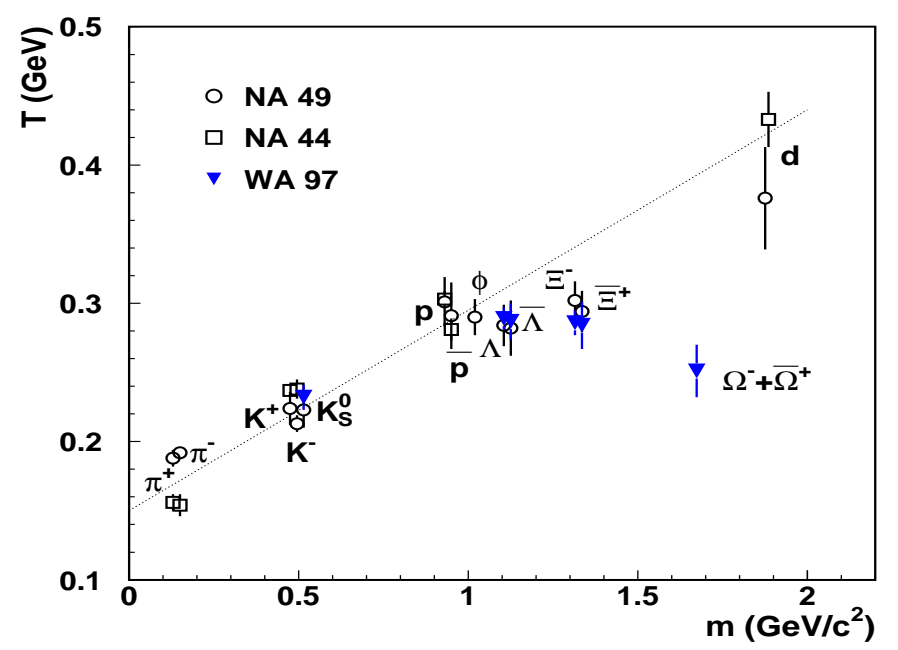

Figure 3. Inverse slopes $T \equiv T_{\text {slope }}$ of the measured $m_{\perp}$-distributions from $158 A \mathrm{GeV} / c \mathrm{~Pb}+\mathrm{Pb}$ collisions for various hadron species, plotted against their rest mass [18]. Results from different experiments are labelled by different symbols. The line is described in [18].

The deuterons don't really belong here either: they are too fragile to be considered part of the equilibrium ensemble. In the thermal state they are continually broken up by collisions, re-forming only at freeze-out by coalescence of protons and neutrons. That they fit into the systematics of Figure 3 is rather a test of the coalescence mechanism by which the deuterons inherit the temperature and flow from their parent nucleons [17]. In fact, the way they fit makes an important statement about the transverse profile of the fireball at freeze-out: for a Gaussian density distribution the deuteron slope should be identical to that of the parent nucleons, whereas the observed larger inverse deuteron slope requires a more box-like density distribution, with more weight at larger flow velocities [17, 19].

An interesting exception to the trend is provided by the $\Omega$ : it's spectrum is considerably steeper. This reflects their earlier kinetic freeze-out, due to an absence of strong scattering resonances with the dominating pion fluid [20] which are essential for the kinetic reequilibration of the other hadron species.

After all these caveats it is clear that a very accurate determination of $\left\langle v_{\perp}\right\rangle$ from the slope in Figure 3 alone is not possible. But transverse flow also affects other observables, in particular two-particle correlations in momentum space, like the quantum statistical BoseEinstein (Hanbury Brown-Twiss) correlations between identical bosons, or the correlations 
due to "soft" final state interactions among the particles after their last "hard" scattering which e.g. cause the coalescence of two nucleons into a deuteron. In both cases correlations occur only between particles which are close in phase-space; by measuring the width of the correlation in momentum space one can thus estimate the size of the emitting source in coordinate space. Collective expansion tends to reduce the size of the regions within which particles can develop such correlations; thermal motion, controlled by the thermal velocity $\sim \sqrt{T / M_{\perp}}$, smears out the flow velocity gradients and thus acts in the opposite direction. This leads to a characteristic dependence of the size of the effective emission region for correlated pairs on their transverse mass $M_{\perp}$; its transverse size is controlled by the transverse flow velocity, $\left\langle v_{\perp}\right\rangle$ as shown by the approximate formula [21,16,17]

$R_{\perp}^{2} \approx \frac{R^{2}}{1+\xi\left\langle v_{\perp}\right\rangle^{2}\left(M_{\perp} / T_{\mathrm{f}}\right)}$

Here $\xi=\mathcal{O}(1)$ is a model-dependent factor which depends on the flow velocity and density profiles, and $R$ characterizes the geometric transverse size of the fireball at freeze-out.

The formula (5) predicts that the effective radius $R_{\perp}$ extracted from the correlations scales only with the transverse mass $M_{\perp}$ of the particle pair, irrespective of the rest masses of the individual particles. This is borne out by experiment, see Figure 4. Similar results, consistent with those shown in Figure 4, were obtained by the NA49 [11] and NA52 collaborations [22].

PbPb Radii versus Transverse Mass

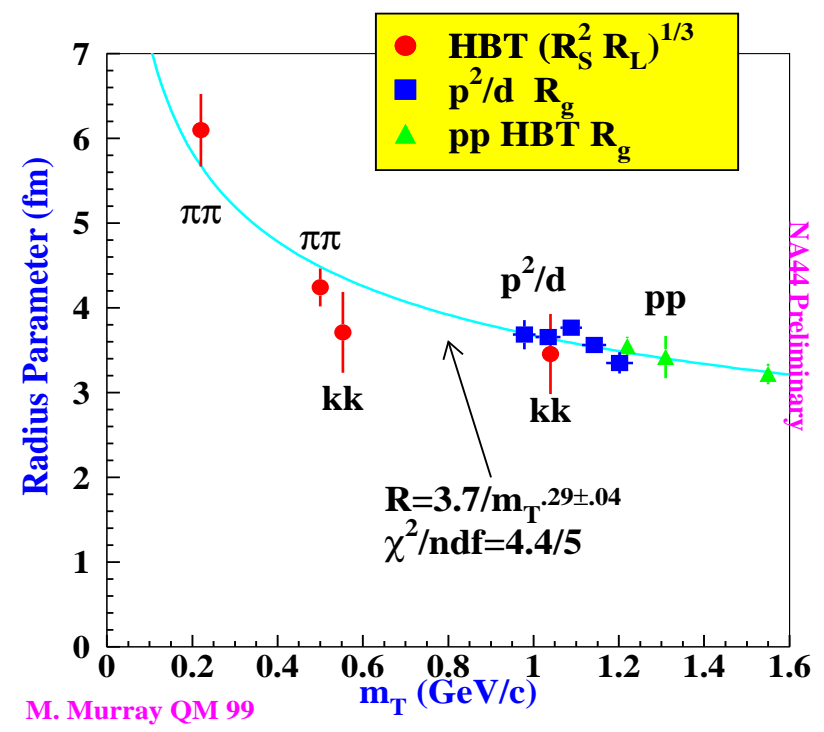

Figure 4. Effective radius $R_{\text {eff }}$ of the emission region extracted from $\pi \pi$ and $K K$ Bose-Einstein (HBT) correlations, from $p p$ final state interaction correlations and from the deuteron coalescence probability $d / p^{2}$, as a function of the transverse mass $M_{T} \equiv$ $M_{\perp}$ of the particle pair. The HBT correlations were analyzed in 3 dimensions, and $R_{\text {eff }}$ was defined by the geometric mean of the two transverse and the longitudinal size parameters, $R_{\text {eff }}=\left(R_{\perp}^{2} R_{\|}\right)^{1 / 3} \equiv\left(R_{s}^{2} R_{L}\right)^{1 / 3}$. (Figure taken from [23.)

While Eq. (5) does not permit to separate $T_{\mathrm{f}}$ from $\left\langle v_{\perp}\right\rangle$ either, the correlation between the two parameters in (5) is exactly opposite to that provided by the spectral slopes in (3) and (4). Combining them in a simultaneous analysis of spectra and correlations [11,24] (see Figure 5) allows for a rather accurate separation of directed collective and random thermal motion, yielding $T_{\mathrm{f}} \approx 100 \mathrm{MeV}$ and $\left\langle v_{\perp}\right\rangle \approx 0.55 \mathrm{c}$. The corresponding thermal energy density is only about $\epsilon_{\mathrm{f}} \approx 0.05 \mathrm{GeV} / \mathrm{fm}^{3}$. Even when the kinetic flow energy is added this still implies an expansion of the fireball volume by a factor 40 from $\tau_{0}$ to $\tau_{\mathrm{f}}$. HBT measurements show that the transverse area grows by a factor $4-5$, consistent with 
the large transverse expansion velocity $\left\langle v_{\perp}\right\rangle$ [24]; the remaing factor 8-10 must come from longitudinal growth.

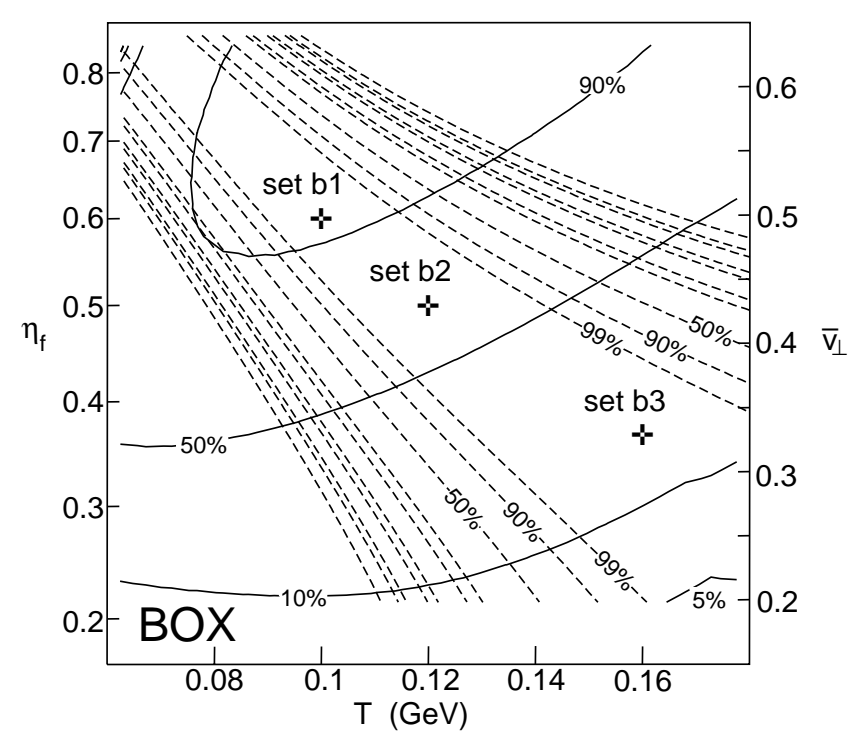

Figure 5. $\chi^{2}$ contours for the fit of the measured HBT radius parameters from $\pi \pi$ Bose-Einstein correlations [11 (widely spaced contours) and of the negative particle spectrum [25] (narrowly spaced contours) in the rapidity window $3.9<Y_{\text {lab }}<4.4$ from $158 A \mathrm{GeV} / c \mathrm{~Pb}+\mathrm{Pb}$ collisions. The best fits require freeze-out temperatures slightly below $100 \mathrm{MeV}$ and average transverse expansion velocities of about $0.55 c$. For details the reader should refer to [24] from where this figure was taken.

\section{THE MISSING RHO: OBSERVING THERMALIZATION AT WORK}

With strong experimental evidence that the Little Bang started out at an energy density above $3 \mathrm{GeV} / \mathrm{fm}^{3}$, but only decoupled at about $50 \mathrm{MeV} / \mathrm{fm}^{3}$, we may ask: how can we find out what happened in between? Here the $\rho$ meson can provide a first answer: it can decay into $e^{+} e^{-}$or $\mu^{+} \mu^{-}$pairs which escape from the fireball without further interactions, and this $\rho$-decay clock ticks at a rate of $1.3 \mathrm{fm} / c$, the natural lifetime of the $\rho$. What I mean by this is that after one generation of $\rho$ 's has decayed, a second generation is created by resonant $\pi \pi$ scattering, which can again decay into dileptons, etc. The number of extra dileptons with the invariant mass of the $\rho$ is thus a measure for the time in which the fireball consists of strongly interacting hadrons [26]. Obviously, $\rho$ mesons do not exist before hadrons appear in the fireball, so they won't tell us anything about a possible QGP phase in its initial stages. But they still allow us to look inside the strongly interacting hadronic fireball at a later stage, still long before the hadrons decouple.

The experimental check of this conjecture yields a surprise: when the CERES/NA45 collaboration looked at the $e^{+} e^{-}$spectrum in $158 \mathrm{AGeV} / c \mathrm{~Pb}+\mathrm{Au}$ collisions (see Figure 6 ), they could not find the $\rho$ at all! Sure, there were extra $e^{+} e^{-}$pairs in the mass region of the $\rho$ and below (about 2.5-3 times as many as expected), but instead of a nice $\rho$-peak at $m_{\rho}=770 \mathrm{MeV}$ one finds only a broad smear [27. Many explanations of the CERES-effect have been proposed, but the simplest one consistent with the data (for a review see [28]) is collision broadening: there is strong rescattering of the pions, not only among each other, but also with the baryons in the hadronic resonance gas, and this modifies their spectral densities and, as a consequence, leads to a smearing of the $\rho$-resonance in the $\pi \pi$ scattering cross section.

This demonstrates that, after first being formed in the hadronization process, the pions (the most abundant species at the SPS) undergo intense rescattering before finally freezing out. And this again is the mechanism which allows the fireball to reach and maintain a 
state of approximate local thermal equilibrium, to build up thermodynamic pressure and to collectively explode, as seen from the above analysis of the freeze-out stage. That the dileptons from collision-broadened $\rho$ 's outnumber those from the decay of unmodified $\rho$ 's emitted at thermal freeze-out (which should show up as a normal $\rho$-peak) shows that the hadronic rescattering stage must have lasted several $\rho$ lifetimes.

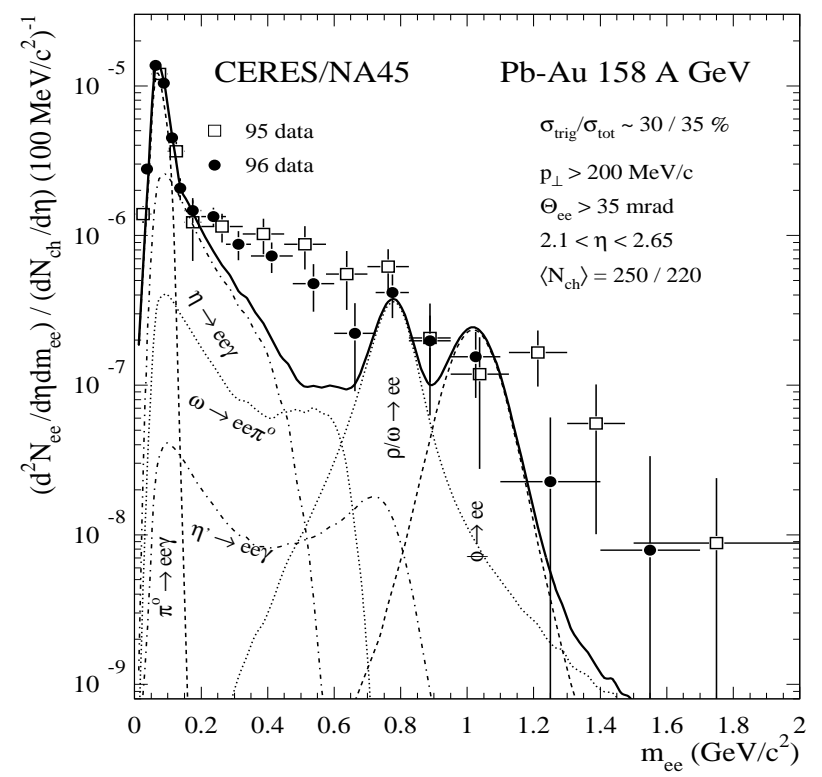

Figure 6. Invariant mass spectrum of $e^{+} e^{-}$pairs from $158 \mathrm{AGeV} / c \mathrm{~Pb}+\mathrm{Au}$ collisions 27. The solid line is the expected spectrum (the sum of the many shown contributions) from the decays of hadrons produced in $p p$ and $p A$ collisions (where it was experimentally confirmed 27]), properly scaled to the $\mathrm{Pb}+\mathrm{Au}$ case. Two sets of data with different analyses are shown. Note that the $\rho$-peak reappears if only $e^{+} e^{-}$pairs with $p_{\perp}>500 \mathrm{MeV} / c$ are selected [27]; such fast $\rho$ 's escape quickly from the fireball and are not as strongly affected by collision broadening.

\section{SEEING THE QUARK-HADRON TRANSITION}

In the rest of this talk I will concentrate on observables which in heavy-ion collisions were found to be drastically different from NN collisions but which we now believe cannot be changed quickly enough by hadronic rescattering during the time available between hadronization and kinetic freeze-out. Observables for which this last property can be firmly established yield insights about where heavy-ion collisions differ from NN collisions already before or during hadronization, irrespective whether or not the hadrons rescatter with each other after being formed.

Of course, the formation of a quark-gluon plasma is one possibility how the early stage of a heavy-ion collision may differ from that in a $\mathrm{NN}$ collision. It is thus important to review a few key QGP predictions and check how they fare in comparison with the data. In the present Section I discuss strangeness enhancement as a QGP signature, returning to two further QGP predictions in the following two Sections.

Strangeness enhancement and chemical equilibration was one of the earliest predicted QGP signatures [29]. The idea is simple: color deconfinement leads to a large gluon density which can create $s \bar{s}$ pairs by gluon fusion, and chiral symmetry restoration makes the strange quarks relatively light, thus reducing the production threshold (not to mention that in the QGP strange quarks can be created without the need for additional light quarks to make a hadron). The two effects together should cause a significant reduction of the time scale for strangeness saturation and chemical equilibration, compared to hadronic rescattering processes after hadronization where the production of strange hadron pairs with opposite strangeness is suppressed by large thresholds and small cross sections. Since 
the production of strange hadrons in $\mathrm{NN}$ and $e^{+} e^{-}$collisions is known to be significantly suppressed relative to the expectation from simple statistical phase-space considerations [30], this should lead to a relative enhancement of strangeness production in heavy-ion collisions.

Kinetic simulations, based on known hadronic properties and interaction cross sections, have now convincingly shown that it is not possible to create a state of hadronic chemical equilibrium and a significant amount of strangeness enhancement out of a non-equilibrium initial state by purely hadronic rescattering (for a recent review see [31]). If you want to get those features out, you have to put them in at the beginning of the simulation.

There may be many different ways of doing so. However, the most efficient way of creating a state of (relative or absolute) hadronic chemical equilibrium appears to be provided by the hadronization process itself: due to color-confinement, the coalescence of colored quarks into hadrons is a process with very large cross sections, allowing for many different arrangements among the quarks with essentially equal probability. If before hadronization the quarks and gluons are essentially uncorrelated (like in a QGP), then the most likely outcome of the hadronization process is a statistical occupation of the hadronic phase-space, i.e. a state of maximum entropy, subject only to the constraints of conservation of energy, baryon number, net strangeness, and (sic!) the total number of available $s \bar{s}$ pairs. Thus, if (as predicted for the QGP [29]) the number of $s \bar{s}$-pairs is enhanced before the onset of hadronization, or by the fragmentation of gluons during hadronization, their statistical distribution over the available hadronic channels will naturally lead to an apparent hadronic chemical equilibrium state (with the appropriate enhancement of, say, the $\bar{\Omega}$ ) even if none of the hadrons ever scattered with each other after being formed.

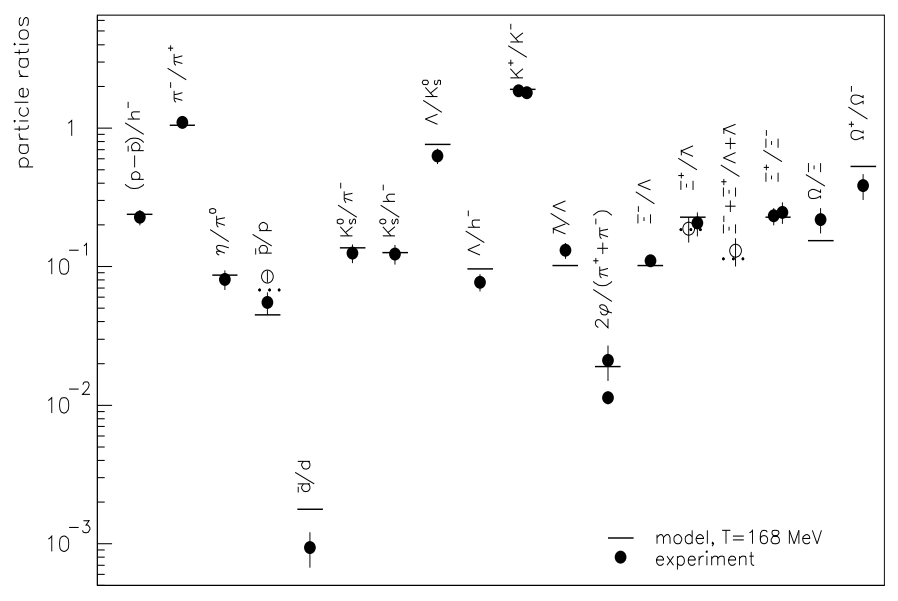

Figure 7. A compilation of measured particle ratios from $158 \mathrm{AGeV} / \mathrm{c}$ $\mathrm{Pb}+\mathrm{Pb}$ collisions, compared with a hadron resonance gas in complete chemical equilibrium (full strangeness saturation) at $T_{\text {chem }}=$ $168 \mathrm{MeV}$ and $\mu_{\mathrm{B}}=266 \mathrm{MeV}$ [32].

Such a state of "apparent" or "pre-established" chemical equilibrium is indeed seen in the experiments: Figure 7 shows some 18 hadronic particle ratios measured in $158 \mathrm{AGeV} / \mathrm{c}$ $\mathrm{Pb}+\mathrm{Pb}$ collisions, compared with a chemical equilibrium fireball model at $T_{\text {chem }}=168 \mathrm{MeV}$ and $\mu_{\mathrm{B}}=266 \mathrm{MeV}$ [32]. The agreement between the data is at least as good as between different experiments. The value of $T_{\text {chem }}$ is interesting: in the maximum entropy sense it characterizes the energy density at which hadronization occurs (about $0.5 \mathrm{GeV} / \mathrm{fm}^{3}$ ) and coincides within errors with the critical temperature for color deconfinement from lattice QCD. If the hadrons were formed by hadronization of a prehadronic state at the critical energy density $\epsilon_{\mathrm{c}}$ and their abundances froze out at $T_{\text {chem }}=168 \mathrm{MeV}$, there was indeed 
no time to achieve this equilibrium configuration by hadronic rescattering; the hadrons must have been "born" into chemical equilibrium [33, 34.

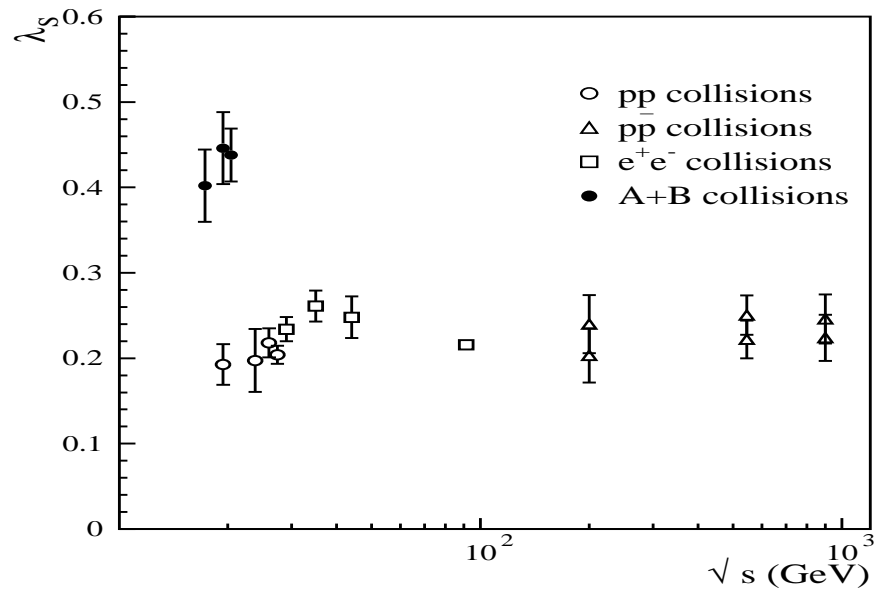

Figure 8. The strangeness suppression factor of produced strange vs. nonstrange valence quarks, $\lambda_{s}=$ $2\langle\bar{s} s\rangle /\left.\langle\bar{u} u+\bar{d} d\rangle\right|_{\text {produced }}$, in elementary particle and heavy-ion collisions as a function of $\sqrt{s}$ [35]. The two points each for $p \bar{p}$ collisions reflect the inclusion (exclusion) of the initial valence quarks.

But this only half the story. Namely, a similar picture of statistical hadronization at the critical energy density $\epsilon_{\mathrm{c}}$ arises even from an analysis of $e^{+} e^{-}, p p$ and $p \bar{p}$ collisions [30]. What is really dramatically different in heavy-ion collisions is the level of strangeness saturation reflected in the apparent chemical equilibrium state: Figure 8 shows that the overall fraction of strange particles is about twice as high in heavy-ion collisions as in elementary particle collisions! Essentially the strangeness suppression observed in NN collisions has disappeared in AA collisions. According to the preceding paragraph, this extra strangeness cannot have been produced by final state rescattering; it thus reflects the properties of the prehadronic state before hadronization. This points to a new, fast strangeness production mechanism before hadronization, as predicted for QGP [29].

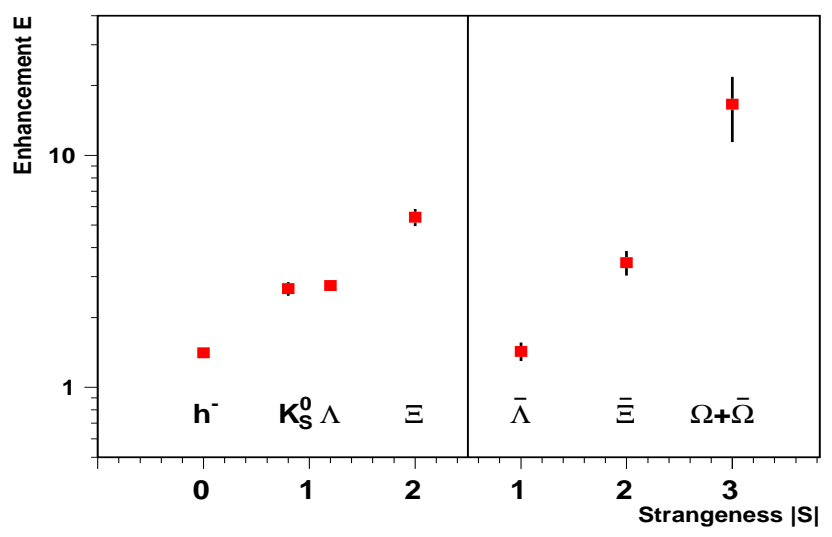

Figure 9. Enhancement factor for the midrapidity yields per participating nucleon in $158 \mathrm{AGeV} / \mathrm{c}$ $\mathrm{Pb}+\mathrm{Pb}$ relative to $\mathrm{p}+\mathrm{Pb}$ collisions for various strange and non-strange hadron species [36].

A very striking way of plotting these findings is shown in Figure 9 [36]: relative to $p+\mathrm{Pb}$ collisions, the number of produced strange hadrons per participating nucleon is the more strongly enhanced the more strange (anti)quarks for its formation are required. For $\Omega$ and $\bar{\Omega}$ this enhancement factor is about 15! The tendency shown in Figure 9 is completely counterintuitive for hadronic rescattering mechanisms, where multistrange (anti)baryons are suppressed by higher thresholds than kaons and $\Lambda$ 's; but it is perfectly consistent with a statistical hadronization picture [37] where multi-strange particles profit more from the global strangeness enhancement than singly strange hadrons.

Kinetic codes like URQMD [38] tend to lose (anti)baryons (and thus a fraction of the 
initial enhancement of multi-strange (anti)baryons) by baryon-antibaryon annihilation during the rescattering stage. It was recently shown [39] that this is to a large extent a manifestation of the lack of detailed balance in the codes which include processes like $\bar{p} p \rightarrow n \pi$ (with $n=5-6$ ) but not their inverse. Rapp and Shuryak 39] argue that, as the system cools below $T_{\text {chem }}$, pions and kaons don't annihilate but instead build up a positive chemical potential which enhances the probability for the inverse reaction and strongly reduces the net annihilation of antibaryons. This is really fortunate, because it is this lack of abundance-changing processes during the hadronic expansion stage which allows us to glimpse the hadronization process itself through the final hadronic abundances, in spite of intense, resonance-mediated elastic rescattering among the hadrons between hadronization at $T_{\text {chem }} \approx 170 \mathrm{MeV}$ and kinetic freeze-out at $T_{\mathrm{f}} \approx 100 \mathrm{MeV}$.

\section{8. $J / \psi$ SUPPRESSION AND COLOR DECONFINEMENT}

The observation of hadronic chemical equilibrium abundances has taken us from kinetic freeze-out all the way back to the hadronization transition. The observed strangeness enhancement gives indirect information about the state that existed before hadronization. It is consistent with the hypothesis that this state consisted of color-deconfined quarkgluon matter and that intense rescattering among the quarks and gluons, before or during the hadronization process, produced the extra strangeness. But this may not be the only explanation. Can one find other, perhaps more direct indications for matter containing deconfined gluons in the early collision stages?

This brings us to the second key prediction for QGP formation: Matsui and Satz suggested [40] that the high gluon density resulting from color deconfinement should Debye-screen the color interaction potential between a $c$ and a $\bar{c}$ quark pair produced during the initial impact of the two nuclei and thus prevent them from binding into charmonium states $\left(J / \psi, \chi_{c}, \psi^{\prime}\right)$. Instead, they would eventually find light quark partners to make hadrons with open charm. This would lead to a suppression of charmonium production in heavy-ion collisions, and the screening mechanism should lead to a specific suppression pattern which, as a function of energy density achieved, first affects the loosely bound $\psi^{\prime}$ and $\chi_{c}$ states and then the strongly bound $J / \psi$ ground state [41].

There is an expected background to this charmonium suppression which already exists in $p A$ collisions and can be studied there in order to extrapolate it to $A A$ : if you put yourself in the CM frame, a $c \bar{c}$ pair created in a hard $p p$ collision (i.e. after $1 /\left(2 m_{c}\right)<$ $0.1 \mathrm{fm} / c$ ) will be affected by interactions with other nucleons from the rest of the nucleus which is still sweeping over it. This "normal suppression" has by now been well studied [41] and is indicated by the straight lines in Figure 10. It follows an exponential attenuation with the length $L$ of cold nuclear matter of density $\rho_{0}$ sweeping over the $c \bar{c}$-pair, with an absorption cross section of about $6 \mathrm{mb}$ for both the weakly bound $\psi$ ' and the tightly bound $J / \psi$ ! The equal absorption is understood as an effect on the pre-resonant $c \bar{c}$ state at early times, before the bound charmonium states actually form (which at SPS energies and above happens only after the whole nucleus has passed over the pair).

Figure 10 shows that a deviation from this "normal" absorption occurs in heavy-ion collisions once the nuclear overlap volume (related to the variable $L$ ) becomes sufficiently large. The weakly bound $\psi$ ' suffers "anomalous absorption" first, at around $L=5 \mathrm{fm}$, 
while for the $J / \psi$ and/or the $\chi_{c}$ (which in $p A$ collisions is known to contribute about $30 \%$ to the measured $J / \psi$ yield via its radiative decay $\chi_{c} \rightarrow \gamma J / \psi$ ) the anomalous absorption does not set in until about $L=7.5 \mathrm{fm}$.
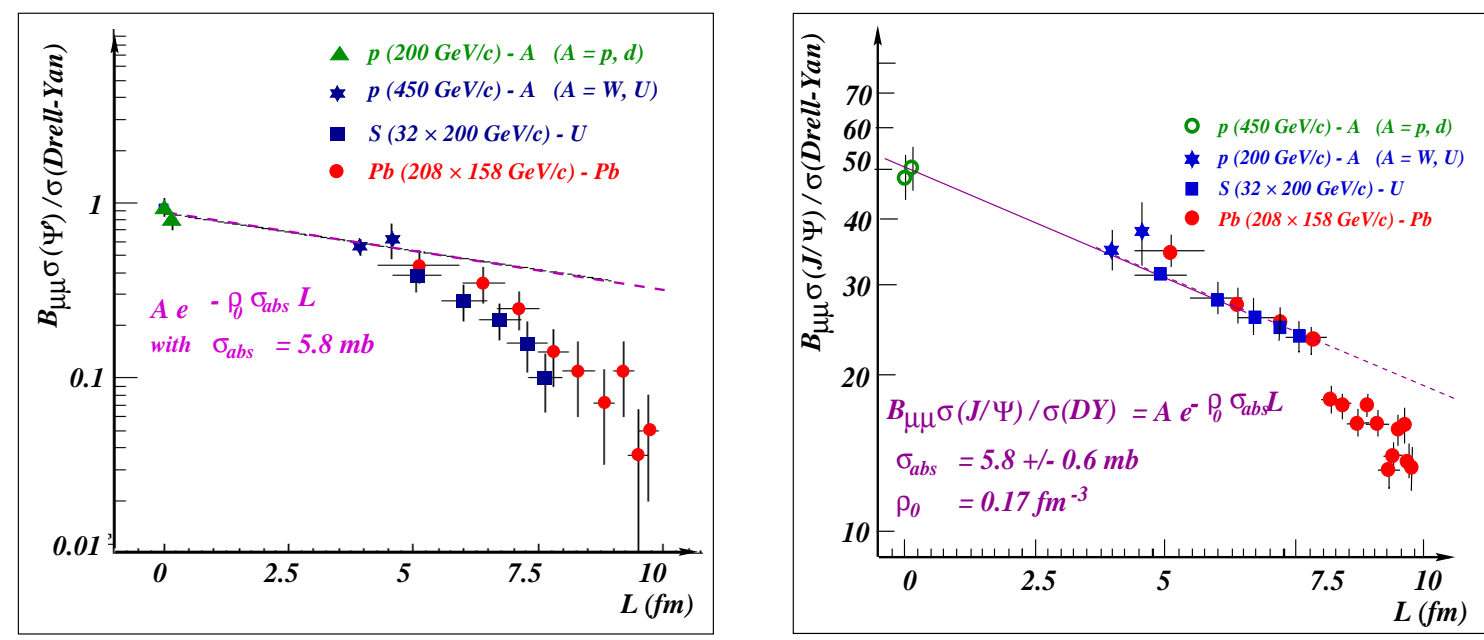

Figure 10. NA38/NA50 data on $\psi^{\prime} / D Y$ and $(J / \psi) / D Y$ [42], plotted as a function of the nuclear thickness parameter $L$ (see text).
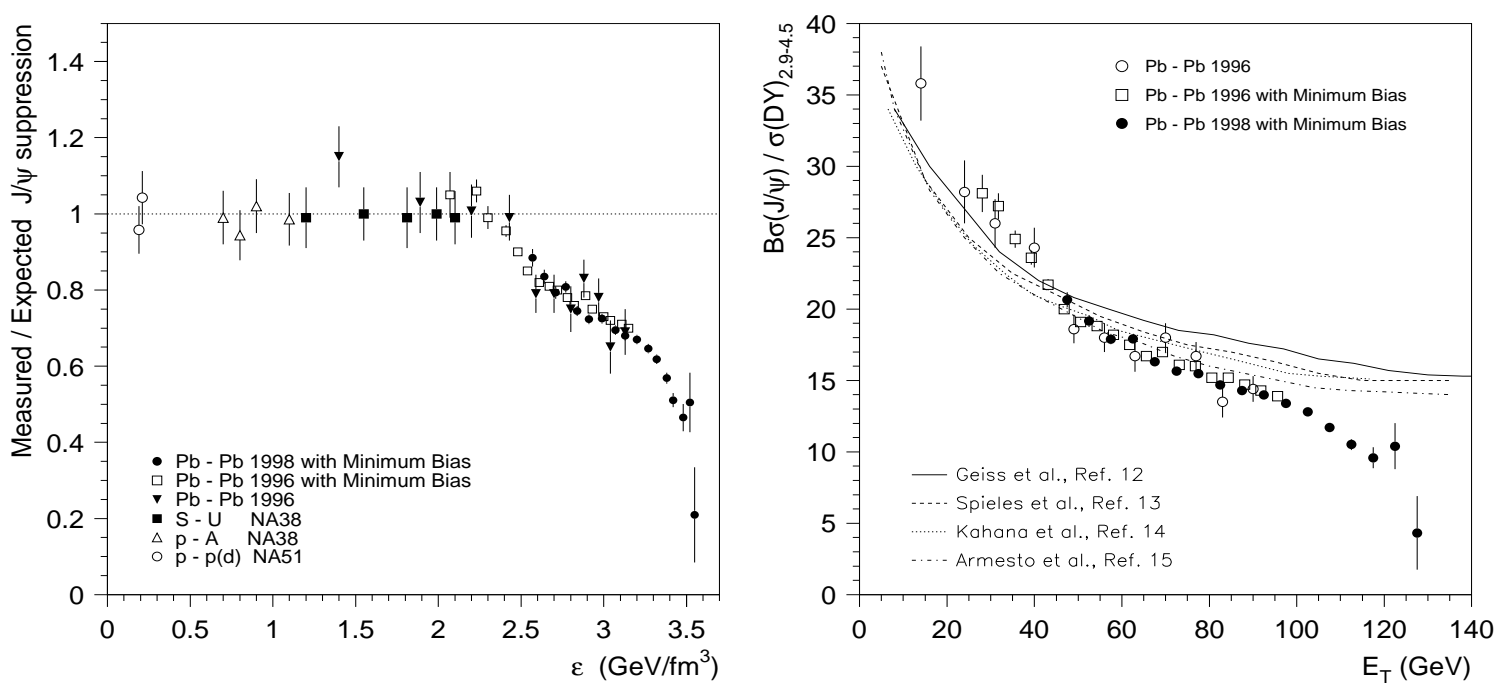

Figure 11. Left: "Anomalous $J / \psi$ suppression" (see text) as a function of initial energy density 43]. Right: The ratio $(J / \psi) /$ Drell-Yan production, as a function of the measured transverse energy $E_{T}$, compared to different hadronic comover models 43.].

The $J / \psi$ suppression has in the meantime been studied in much greater detail as shown in Figure 11. The left part shows the "anomalous suppression" (with the normal preresonant absorption on the incoming nuclei divided out) as a function of energy density [43]. The latter is computed from the measured transverse energy $E_{T}$ via Bjorken's formula (11), where the effective overlap area in the denominator is related to $E_{T}$ via a geometric model and Glauber theory [43]. The observed suppression pattern is interesting: it occurs in two "waves", with an intermediate flattening after about $30 \%$ of suppression (which one might be tempted to associate with the complete suppression of the $\chi_{c}$ component) followed by a stronger suppression at energy densities above $3 \mathrm{GeV} / \mathrm{fm}^{3}$. 
The real origin of this "wavy" structure is not yet clear; on a superficial level it is qualitatively consistent with a suppression hierarchy $\psi^{\prime} \rightarrow \chi_{c} \rightarrow J / \psi$ as expected from the QGP color-screening scenario. In any case, the right part of Figure 11 shows that it can not be reproduced by conventional hadronic final state interactions (collision dissociation) between the charmonium and the produced hadrons. Such "hadronic comover models" must be tuned to relatively large inelastic $J / \psi$-comover scattering cross sections to even reproduce the average suppression in $\mathrm{Pb}+\mathrm{Pb}$ collisions, at the expense of sometimes overpredicting the effect in $\mathrm{S}+\mathrm{U}$ collisions. Even then most of the destructive interactions happen at very early times where the comover densities are so high that a hadronic description should really not yet apply. Most importantly, however, they all consistently fail to reproduce the strong suppression at very high $E_{T}$.

It was recently noted that the apparent onset of the second, stronger suppression above $\epsilon=3 \mathrm{GeV} / \mathrm{fm}^{3}$ coincides roughly with the "knee" in the $E_{T}$-distribution, i.e. with the point of full nuclear overlap above which one enters the region of $E_{T}$ fluctuations 44,45. These fluctuations tend to increase the $J / \psi$ suppression even in the comover models, but the effect is not strong enough to reproduce the data 44. On the other hand, if one associates them with fluctuations of the energy density in the QGP Debye-screening approach the fluctuation effects are much stronger and the data can be reproduced 45. It would be important to clarify this issue, e.g. by performing $U+U$ collisions where, due to the deformation of the uranium nucleus, in the tip-on-tip configuration similar energy densities can be reached without having to exploit $E_{T}$ fluctuations.

\section{THERMAL ELECTROMAGNETIC RADIATION}

The third (and earliest) key prediction for QGP formation was thermal radiation of (real and virtual) photons from the thermalized quarks in the QGP 46. They are conceptually clean direct probes of the QGP phase itself, but experimentally difficult due to large backgrounds. Nonetheless, experiments at the SPS have searched for this type of radiation. An excess yield in the photon spectrum above about $1.5 \mathrm{GeV}$ which is attributed to direct emission was found by WA98 [47] and discussed by Th. Peitzmann at this meeting. It is, I think, fair to say that the relation of this signal with thermal QGP radiation is presently unclear. The NA50 Collaboration found an excess in the dimuon spectrum between the $\phi$ and $J / \psi$ peaks which was interpreted by them as an excess in open charm production [48] but which may also be due to thermal QGP radiation [49]. Again, the true origin is not clear. The situation is such that, for the initial temperatures reachable at the SPS, the theoretical predictions for a thermal radiation signal are so low that it is not obvious that it can be dug out from the experimental background. Since the thermal radiation rate goes with $T^{4}$, the higher initial temperatures reachable at RHIC and LHC, combined with the longer lifetime of the plasma phase, should help a lot to see the plasma "shine" and thus confirm this prediction experimentally.

\section{CONCLUSIONS}

Relativistic heavy-ion collisions at the CERN SPS have taken us into new and unprecedented regions of energy density: in $\mathrm{Pb}+\mathrm{Pb}$ collisions at $158 \mathrm{AGeV} / \mathrm{c}$ initial energy densities of about $3.5 \mathrm{GeV} / \mathrm{fm}^{3}$ (i.e. more than 20 times the energy density of cold nuclear 
matter) have been created over large volumes. If the matter was approximately thermalized even at this early stage (for which we do not yet have convincing direct evidence, although data on elliptic flow [50,51], which I had no time and space to discuss, point in this direction), the initial temperature was around $210-220 \mathrm{MeV}$, i.e. $30 \%$ above the critical temperature for color deconfinement.

We have strong and direct experimental evidence for a large degree of thermalization and strong collective behaviour in the late stages of the collision, driven by intense rescattering among the fireball constituents which is directly visible in the low-mass dilepton spectra. At kinetic freeze-out the fireball radiates hadrons with a temperature of about $100 \mathrm{MeV}$, at the same time undergoing collective explosion with more than half the light velocity (the "Little Bang").

Extensive theoretical simulations have shown that conventional hadronic processes during the hadronic rescattering phase lead mostly to elastic collisions and are very inefficient in changing the final hadron abundances. The observed hadronic particle ratios thus reflect the "primordial hadrosynthesis" in the Little Bang and provide a direct glimpse of the hadron formation stage. The data show that the hadrons are born into a state of "pre-established chemical equilibrium" at a temperature of about $170 \mathrm{MeV}$ which coincides with the deconfinement temperature predicted by lattice QCD. This is the first observation of thermal equilibrium matter at such a high temperature and energy density. The phenomenon is most easily understood in terms of statistical hadronzation of a QGP, although other mechanisms with similar statistical features cannot be excluded. The experimentally determined "chemical" and "thermal" freeze-out points can be connected by an isentropic expansion trajectory as indicated in Figure 1. (Note that the part of the same trajectory which runs through the QGP phase is so far speculative.)

Strangeness enhancement and charmonium suppression have been predicted as QGP signatures and are indeed found to characterize the prehadronic state from which the observed hadrons appear during hadronization. These features cannot be understood in terms of conventional hadronic final state rescattering effects after hadronization. They are consistent with QGP expectations, although other explanations may still be possible. The inability to understand these properties in terms of known hadronic physics warrants the characterization of this prehadronic state as a "new state of matter" [1].

So what is missing to claim "discovery" of the quark-gluon plasma? First, on the theoretical side, we only know that with known hadronic physics we can not describe the data, but it has not yet been shown that a fully dynamical theory which begins with QGP and follows the system until freeze-out actually can describe all observations. One reason is that a description of strongly interacting matter and its dynamics in the neighborhood of the phase transition is an exceedingly difficult problem, and that at the SPS we are never far away from the phase transition. This task may thus become easier at RHIC/LHC than at the SPS. Furthermore, the evolution of the "late signatures" from $p p$ via $p A$ to $A A$ collisions, to establish a clear line between "conventional hadronic physics" and "new physics" has so far not received enough careful theoretical attention. This should be remedied, but it requires much improved $p p$ and $p A$ data at the same energy, with more differential experimental information. Such data can (only) be obtained at the SPS.

Further important experimental questions which can be answered at the SPS (and in a few cases only there) are: Assuming that we have seen quark deconfinement, where is 
its energy threshold? How big does the collision system have to be to establish approximate thermal equilibrium and strangeness saturation and to exhibit collective flow? If charmonia are suppressed, what happens with open charm - is Drell-Yan production of dileptons (which depends on the quark structure functions in the colliding nuclei) really the appropriate normalization for $J / \psi$ suppression (which is sensitive to the gluon structure functions since $c \bar{c}$ pairs are made from gluons)? Some answers will be provided by data already collected at lower beam energies and with smaller nuclei and more peripheral collisions. A search for open charm at the SPS was proposed [52] and recently approved. But other questions are still waiting for proposals to be made.

Still, with the SPS we will never be able to get "away from the edge". A detailed characterization of the "new state of matter" will only be possible when the larger initial energy densities and resulting longer plasma lifetimes before hadronization provided by RHIC and LHC become available. The higher initial temperatures are expected to give an observable thermal radiation signal, thus allowing to measure the thermal "QGP structure functions". The higher collision energies allow for the creation of high- $p_{t}$ jets which can then be used as probes of the QGP, by their interactions with the plasma when they penetrate it. And last not least, the fact that RHIC is a dedicated heavy-ion machine at which experiments can be run for a large fraction of each year is conducive to the systematic studies that will be required to eventually obtain a complete and consistent picture of quark-gluon plasma dynamics.

\section{REFERENCES}

1. CERN Press Release Feb. 10, 2000: http://cern.web.cern.ch/CERN/Announcements/2000/NewStateMatter/

2. U. Heinz and M. Jacob, nucl-th/0002042.

3. Proceedings of "Quark Matter '97", T. Hatsuda et al. (Eds.), Nucl. Phys. A 638 (1998); of "Strangeness in Quark Matter 1998", M. Morando (Ed.), J. Phys. G 25 (1999); and of "Quark Matter '99", L. Riccati et al. (Eds.), Nucl. Phys. A 661 (1999).

4. http://webcast.cern.ch/Archive/2000/2000-02-10.

5. For a recent discussion of detailed features of the QCD phase diagram see K. Rajagopal, Comments on Modern Physics (Part A: Comm. Nucl. Part. Phys.) 2 (2000), in press (hep-ph/0009058).

6. F. Karsch, Nucl. Phys. B (Proc. Suppl.) 83-84 (2000) 14.

7. F. Karsch, private communication, based on work presented in F. Karsch, E. Laermann, and A. Peikert, Nucl. Phys. B (Proc. Suppl.) 83-84 (2000) 390; Phys. Lett. B 478 (2000) 447; and in A. Peikert's PhD thesis (unpublished).

8. F. Karsch and E. Laermann, Phys. Rev. D 50 (1994) 6954.

9. S.A. Bass, M. Gyulassy, H. Stöcker, and W. Greiner, J. Phys. G 25 (1999) R1.

10. J.D. Bjorken, Phys. Rev. D 27 (1983) 140.

11. NA49 Collaboration, H. Appelshäuser et al., Eur. Phys. J. C 2 (1998) 661.

12. NA49 Collaboration, T. Alber et al., Phys. Rev. Lett. 75 (1995) 3814; WA98 Collaboration, M. Aggarwal et al., Nucl. Phys. A610 (1996) 200c; and nucl-ex/0008004.

13. U. Heinz, nucl-th/9710065.

14. H. Weber et al., Phys. Lett. B 442 (1998) 443. 
15. K.S. Lee, U. Heinz, and E. Schnedermann, Z. Phys. C 48 (1990) 525.

16. T. Csörgö, B. Lörstad, Nucl. Phys. A 590 (1995) 465c; Phys. Rev. C 54 (1996) 1390.

17. R. Scheibl and U. Heinz, Phys. Rev. C 59 (1999) 1585.

18. WA97 Collaboration, F. Antinori et al., Eur. Phys. J. C 14 (2000) 633.

19. A. Polleri, J.P. Bondorf, and I.N. Mishustin, Phys. Lett. B 419 (1998) 19.

20. H. van Hecke, H. Sorge, and N. Xu, Phys. Rev. Lett. 81 (1998) 5764.

21. S. Chapman, J.R. Nix, and U. Heinz, Phys. Rev. C 52 (1995) 2694.

22. NA52 Collaboration, G. Ambrosini et al., New J. Phys. 1 (1999) 22.

23. NA44 Collaboration, M. Murray et al., Nucl. Phys. A 661 (1999) 456c.

24. B. Tomášik, U.A. Wiedemann, and U. Heinz, nucl-th/9907096.

25. NA49 Collaboration, P.G. Jones et al., Nucl. Phys. A 610 (1996) 188c.

26. U. Heinz and K.S. Lee, Phys. Lett. B 259 (1991) 162.

27. CERES Collaboration, B. Lenkeit et al., Nucl. Phys. A661 (1999) 23c.

28. R. Rapp and J. Wambach, hep-ph/9909229, Adv. Nucl. Phys., in press.

29. J. Rafelski and B. Müller, Phys. Rev. Lett. 48 (1982) 1066; P. Koch, B. Müller, and J. Rafelski, Phys. Rep. 142 (1986) 167.

30. F. Becattini, Z. Phys. C 69 (1996) 485; F. Becattini and U. Heinz, ibid. 76 (1997) 269.

31. U. Heinz, Nucl. Phys. A 661 (1999) 140c.

32. P. Braun-Munzinger, I. Heppe, and J. Stachel, Phys. Lett. B 465 (1999) 15.

33. U. Heinz, Nucl. Phys. A 638 (1998) 357c; J. Phys. G 25 (1999) 263; hep-ph/9902424.

34. R. Stock, Prog. Part. Nucl. Phys. 42 (1999) 295; Phys. Lett. B 456 (1999) 277.

35. F. Becattini, M. Gaździcki, and J. Sollfrank, Eur. Phys. J. C 5 (1998) 143.

36. WA97 Collaboration, R. Lietava et al., J. Phys. G 25 (1999) 181 (see p 460 in the same volume for the correct Fig. 7!).

37. J. Rafelski, Phys. Lett. B 262 (1991) 333; A. Bialas, Phys. Lett. B 442 (1998) 449.

38. S.A. Bass et al., Phys. Rev. C 60 (1999) 021902.

39. R. Rapp and E.V. Shuryak, hep-ph/0008326.

40. T. Matsui and H. Satz, Phys. Lett. B 178 (1986) 416.

41. For a recent review see H. Satz, Rep. Prog. Phys. 63 (2000) 1511.

42. NA50 Collaboration, L. Ramello et al., Nucl. Phys. A 638 (1998) 261c; M. Abreu et al., Phys. Lett. B 450 (1999) 456.

43. NA50 Collaboration, M. Abreu et al., Phys. Lett. B 477 (2000) 28.

44. A. Capella, E.G. Ferreiro, and A.B. Kaidalov, Phys. Rev. Lett. 85 (2000) 2080.

45. J.-P. Blaizot, P.M. Dinh, and J.-Y. Ollitrault, nucl-th/0007020.

46. E.V. Shuryak, Phys. Lett. 78 B (1978) 150; K. Kajantie and H.I. Miettinen, Z. Phys. C 9 (1981) 341.

47. WA98 Collaboration, M.M.Aggarwal et al. nucl-ex/0006007 and nucl-ex/0006008.

48. NA38 and NA50 Collaborations, M.C. Abreu et al., Eur. Phys. J. C 14 (2000) 443.

49. R. Rapp and E.V. Shuryak, Phys. Lett. B 473 (2000) 13; K. Gallmeister, B. Kämpfer, and O.P. Pavlenko, ibid., p. 20.

50. NA49 Collaboration, H. Appelshäuser et al., Phys. Rev. Lett. 80 (1998) 4136; A.M. Poskanzer, S.A. Voloshin et al., Nucl. Phys. A 661 (1999) 341c.

51. P. Kolb, J. Sollfrank, U. Heinz, Phys. Lett. B 459 (1999) 667; and hep-ph/0006129.

52. Study of prompt dimuon and charm production with proton and heavy ion beams at the CERN SPS, CERN/SPSC 2000-010 